\title{
Medical Internet of Things and Big Data in Healthcare
}

\author{
Dimiter V. Dimitrov, MD, PhD \\ Diavita Ltd., Varna, Bulgaria
}

Objectives: A number of technologies can reduce overall costs for the prevention or management of chronic illnesses. These include devices that constantly monitor health indicators, devices that auto-administer therapies, or devices that track realtime health data when a patient self-administers a therapy. Because they have increased access to high-speed Internet and smartphones, many patients have started to use mobile applications (apps) to manage various health needs. These devices and mobile apps are now increasingly used and integrated with telemedicine and telehealth via the medical Internet of Things (mIoT). This paper reviews mIoT and big data in healthcare fields. Methods: mIoT is a critical piece of the digital transformation of healthcare, as it allows new business models to emerge and enables changes in work processes, productivity improvements, cost containment and enhanced customer experiences. Results: Wearables and mobile apps today support fitness, health education, symptom tracking, and collaborative disease management and care coordination. All those platform analytics can raise the relevancy of data interpretations, reducing the amount of time that end users spend piecing together data outputs. Insights gained from big data analysis will drive the digital disruption of the healthcare world, business processes and real-time decision-making. Conclusions: A new category of "personalised preventative health coaches" (Digital Health Advisors) will emerge. These workers will possess the skills and the ability to interpret and understand health and well-being data. They will help their clients avoid chronic and diet-related illness, improve cognitive function, achieve improved mental health and achieve improved lifestyles overall. As the global population ages, such roles will become increasingly important.

Keywords: Telemedicine, Smartphone, Mobile Applications, Wireless Technology, Disease Management

\section{Introduction}

The Internet of Things (IoT) is a network of physical devices and other items, embedded with electronics, software, sen-

Submitted: June 29, 2016

Accepted: July 18, 2016

\section{Corresponding Author}

Dimiter V. Dimitrov, MD, PhD

Diavita Ltd., Varna, Bulgaria. E-mail: dimiter.v. dimitrov@gmail.com

This is an Open Access article distributed under the terms of the Creative Commons Attribution Non-Commercial License (http://creativecommons.org/licenses/by$\mathrm{nc} / 4.0 /$ ) which permits unrestricted non-commercial use, distribution, and reproduction in any medium, provided the original work is properly cited.

(C) 2016 The Korean Society of Medical Informatics sors, and network connectivity, which enables these objects to collect and exchange data [1]. Its impact on medicine will be perhaps the most important, and personal, effect. By 2020, $40 \%$ of IoT-related technology will be health-related, more than any other category, making up a $\$ 117$ billion market [2]. The convergence of medicine and information technologies, such as medical informatics, will transform healthcare as we know it, curbing costs, reducing inefficiencies, and saving lives.

Figure 1 illustrates how this revolution in medicine will look in a typical IoT hospital, in practice. A patient with diabetes will have an ID card that, when scanned, links to a secure cloud which stores their electronic health record vitals and lab results, medical and prescription histories. Physicians and nurses can easily access this record on a tablet or 


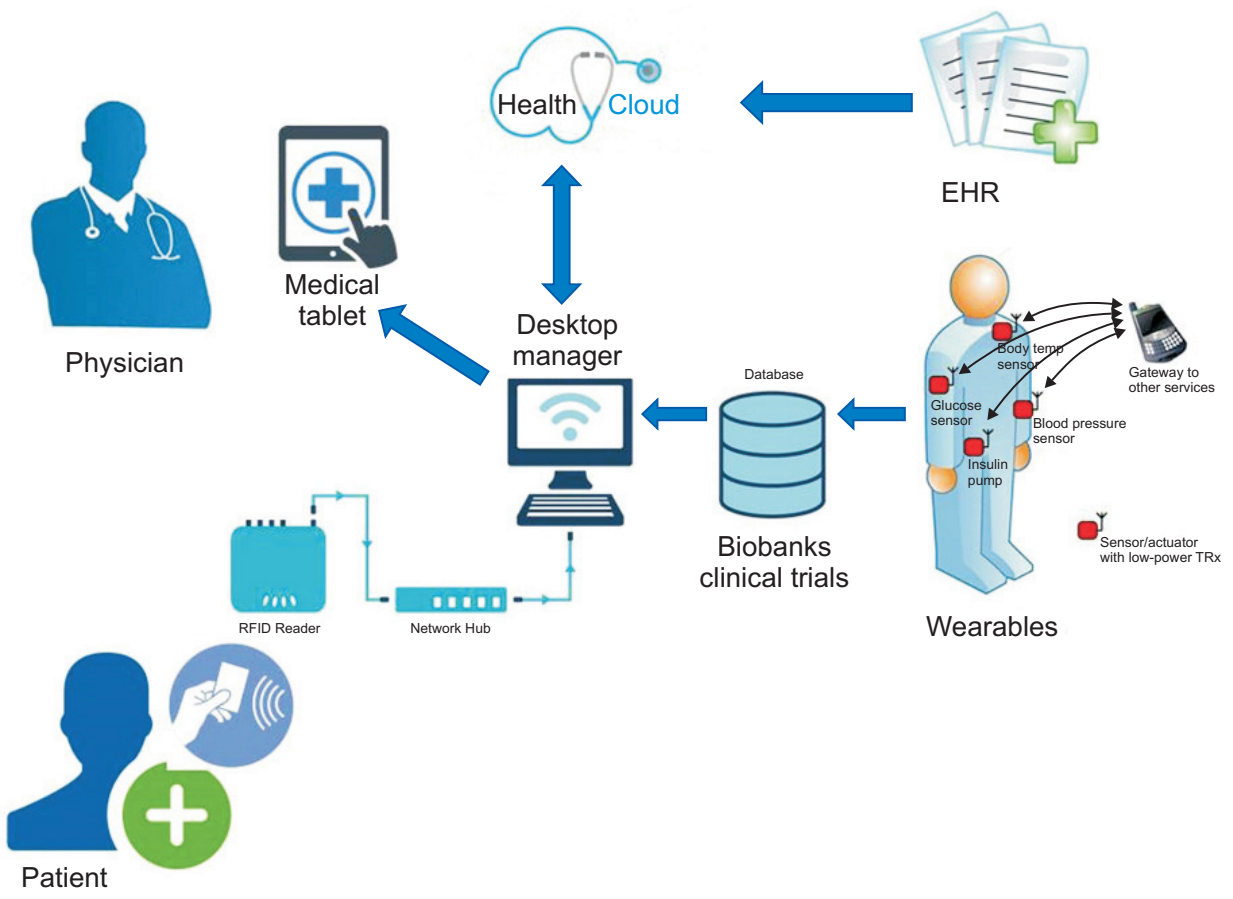

Figure 1. An illustration of how this revolution in medicine will look in a typical Internet of Things (IoT) hospital, in practice. desktop computer.

It sounds pretty basic, but the adoption of Electronic Health Records (EHRs) is a game changer. In less than a decade, an ink-and-paper system of managing records that goes back thousands of years will be digitized and replaced [3]. The advantages are obvious and many. Paper records, often written in questionable penmanship, can get stuffed away in filing cabinets, out of the reach of researchers or other healthcare providers. Instead, by keeping all the important information in one place, and easily sharable, EHRs will eliminate many inefficiencies, and save lives.

One of the major challenges to implementing the IoT has to do with communication; although many devices now have sensors to collect data, they often talk with the server in their own language. Manufacturers each have their own proprietary protocols, which means sensors by different makers can't necessarily speak with each other. This fragmented software environment, coupled with privacy concerns and the bureaucratic tendency to hoard all collected information, frequently maroons valuable info on data islands, undermining the whole idea of the IoT.

Precision medicine, as it's called, is a term that will be frequently heard in coming years [4]. It begins with genomics and goes through the rest of the omics platforms, providing multiscale data for analysis and interpretation [5]. In 2015, Intel and the Oregon Health and Science University launched a joint project, the Collaborative Cancer Cloud: a high-performance analytics platform that collects and securely stores private medical data that can be used for cancer research. Though the platform began with cancer, Intel intends to open up the federated cloud network to other institutions, including ones working on cures to diseases like Parkinson's.

Engineering simulation solutions are making medicine participatory, personalized, predictive and preventive ( $\mathrm{P} 4$ medicine) via the medical Internet of Things (mIoT) [6].

\section{IoT - The Future of Pharma?}

Pharma companies long ago realized that just selling traditional medicines will not produce growth nor even sustain competitiveness. This fundamental change, known as moving 'beyond the pill', typically arises from one or two realizations: (1) medicines alone are often not enough to achieve optimal clinical outcomes for patients, and (2) as pharmaceutical pipelines dry up, 'beyond-the-pill' businesses can be valuable new sources of revenues. This has created growing interest in methods of utilizing the new technologies and business processes for development and patient care, leading to Pharma IoT.

The Pharma IoT concept involves digitalization of medical products and related care processes using smart connected medical devices and IT services (web, mobile, apps, etc.) during drug development, clinical trials and patient care. The outcomes of Pharma IoT in development and clinical trials can employ combinations of advanced technologies 
and services to create totally new kinds of disease treatment possibilities (e.g., Treatment 2.0).

In patient care, Pharma IoT will enable patients and healthcare professionals to use medicines with advanced sensor hardware, and craft personalized care services and processes (Product 2.0). Good examples of the Pharma IoT solutions are the connected sensor wearables for Parkinson's disease and multiple sclerosis patients, which provide medication management, improving the patient outcomes and the quality of life [7].

In addition, existing medical device products such as inhalers and insulin pens can be added to the sensor and connectivity technologies to collect data for further care analytics, and even personalized therapy [8]. All this will substantially improve personal medication and care processes, because patient care data provides new sources of innovation and competitiveness.

The transformation also involves some challenges: at the same time, pharma companies need to take into account the forthcoming European Union (EU) data protection and privacy legislation, which will give patients control of their care data [9]. For example, patients will be allowed to transfer their care and health data across multiple service providers, leading to the emergence of totally new kinds of service platforms and business models, e.g., data brokers [10].

\section{Devices and Mobile Apps for Healthcare}

We are heading into the age of information, where knowledge and data will be key. We are also entering the age of the customer, in which more than ever the customer is going to determine what they want. myTomorrows is one example of the changing look of business models, in this case, directly connecting customers and pharma [11].

In this new age, devices and apps will be used to create a "health selfie". For example:

- The Myo, originally a motion controller for games, is now being used in orthopedics for patients who need to exercise after a fracture. With the aid of the Myo, patients can monitor their progress and doctors can measure the angle of movement.

- The Zio Patch measures heart rate and electrocardiogram (ECG) and is the US Food and Drug Administration approved [12].

Where is pharma in all this turmoil? Interestingly, there are signs that pharma is reaching out from its traditional medicine-centric approach.
- Glaxo recently announced that it is investing in electroceuticals, bioelectrical drugs that work by micro-stimulation of nerves [13].

- J\&J has teamed up with Google to develop robotic surgery. In addition, they are collaborating with Philips on wearable devices such as blood pressure monitors [14].

- Novartis is working with Google (again) on sensor technologies, such as the smart lens, and a wearable device to measure blood glucose levels [15].

Sensors can provide a lot of information to support pharma development, but it is particularly important to recruit the right patients for the right clinical trials. Body sensors, once gadgets that were mainly used by athletes and runners, are now rapidly entering the general market, and consumers and pharma will soon have access to a wealth of information including not only pulse, blood pressure, ECG and respiratory rate, but also more advanced data, such as inflammation, sleep patterns, etc.

A number of mobile apps which support device handling have emerged, including myDario and SleepBot among others $[16,17]$. The Hacking Medicine Institute recently announced RANKED Health, a program to critically evaluate and rank health-focused applications and connected devices [18].

It has been predicted that in the near future we will look at our phone or smart watch to check health outcomes more often than we do now to check our mail or WhatsApp. A typical situation might involve an elderly person, recovering from a medical condition at home, linked to a combination of several connected services streaming data towards different parties, such as family members, tele-carer and physicians (Figure 2).

Recently it was announced that Medtronic will be partnering with a digital health app company named Canary Health to be a re-seller of its digital chronic disease management programs, including its CDC-recognized Diabetes Prevention Program, which is aimed at changing behaviors in prediabetic people. But the partnership goes beyond just reselling Canary Health's digital tools. In fact, both Canary Health and Medtronic plan to develop solutions that "leverage Medtronic's devices, services and infrastructure as well as Canary Health's suite of behavior-change programs, design expertise, and deep user engagement experience," according to a Canary Health news release [19].

One reason that Medtronic must have been attracted to Canary Health is that the company's digital tools are reimbursable. As digital health programs mature, payers are looking at innovative, yet proven, ways to reduce their cost burden 


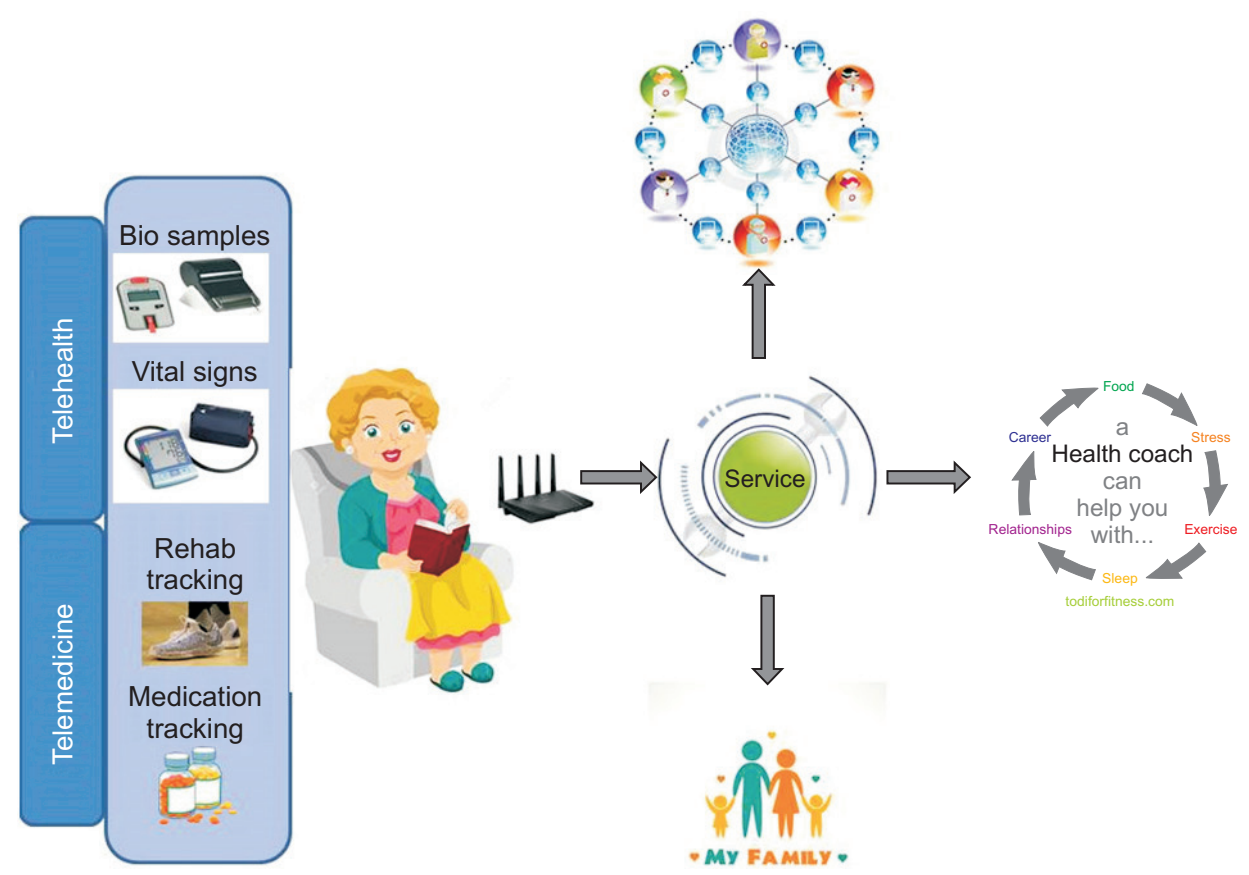

Figure 2. A typical situation involved an elderly person, recovering from a medical condition at home, linked to a combination of several connected services streaming data towards different parties, such as family members, tele-carer and physicians.

for chronic diseases like diabetes.

According to the Centers for Disease Control and Prevention (CDC), people with prediabetes who take part in a structured lifestyle change program-like the one Canary Health has developed, or programs championed by Omada Health and Noom Health, among others- "can cut their risk of developing type 2 diabetes by 58\% (71\% for people over 60 years old)" [20]. The CDC adds that "this finding was the result of a program helping people lose $5 \%$ to $7 \%$ of their body weight through healthier eating and 150 minutes of physical activity a week" [21].

Given that diabetes is an expensive, chronic disease, hospitals, doctors, patients, and payers are equally keen to tame this epidemic. In other words, the move is helping to transform companies from simply providing care to the sick to actually delivering healthcare.

\section{Data, Data, Data}

The driver behind all these wearable sensors is the data that is generated, and various parties are trying to bundle the data streams and obtain control. Microsoft developed the Health Vault, an e-health safe, acting as an EMR. In Holland the Radboud University Medical Center collaborated with Philips and Salesforce on HereIsMyData, a database where patients can store their health data and determine who can access them [22]. The role of Salesforce is interesting. The Salesforce platform powers Veeva, the customer relationship management (CRM) now widely used in pharma. This posi- tions Salesforce to be able to bridge the gap between patient's medical data and pharma.

"Big data" is a phrase that has been used pervasively by the media and the lay public in the last several years. While many definitions have been proposed, the common denominator seems to include the "three V's"-Volume (vast amounts of data), Variety (significant heterogeneity in the type of data available in the set), and Velocity (speed at which a data scientist or user can access and analyze the data) [23].

Defined as such, healthcare has become one of the key emerging users of big data. For example, Fitbit and Apple's ResearchKit can provide researchers access to vast stores of biometric data on users, which can then be used to test hypotheses on nutrition, fitness, disease progression, treatment success, and the like.

Most complex high dimensional data sets include imaging (photos, X-rays, MRIs, and slides), wave analysis such as EEG and ECG, audio files with associated transcripts, free text notes with natural language processing (NLP) outputs, and mappings between structured concepts such as lab tests and the Logical Observation Identifiers Names and Codes (LOINC) codes or the International Classification of Diseases-9 (ICD9) and ICD10 codes. Among the things that the data analysis should provide is the means to continuously update the annotations based on acquired knowledge, while keeping the location of the data in place.

The Centers for Medicare \& Medicaid Services (CMS) have vast stores of billing data that can be mined to promote high 
value care; the same is true of private health insurers. And hospitals have attempted to reduce re-admission rates by targeting patients where predictive artificial intelligence (AI) algorithms indicate people who may be at highest risk based on an analysis of available data collected from existing patient records (Figure 3).

Underlying these and many other potential uses, however, are a series of technology, legal and ethical challenges relating to, among other things, privacy, discrimination, intellectual property, tort, and informed consent, as well as research and clinical ethics [24].

\section{Challenges for mloT}

Leading IoT platforms must provide simple, powerful application access to IoT devices and data to help designers rapidly compose analytics applications, visualization dashboards and mIoT apps. The following are 5 key capabilities that leading platforms must enable:

(1) Simple connectivity: A good IoT platform makes it easy to connect devices and perform device management functions, scaled through cloud-based services, and to apply analytics to gain insight and achieve organizational transformation.

(2) Easy device management: A thoughtful approach to device management enables improved asset availability, increased throughput, minimized unplanned outages and reduced maintenance costs.

(3) Information ingestion: Intelligently transform and store IoT data. APIs bridge the divide between the data and the cloud, making it easy to pull in the data that's needed. Data is ingested from diverse data sources and platforms, then the essential values are extracted using rich analytics.

(4) Informative analytics: Gain insight from huge volumes of IoT data to make better decisions and optimize operations. Apply real-time analytics to monitor current conditions and respond accordingly. Leverage cognitive analytics with both structured and unstructured data to understand situations, reason through options, and learn as conditions change. An intuitive dashboard makes it all easy to under-

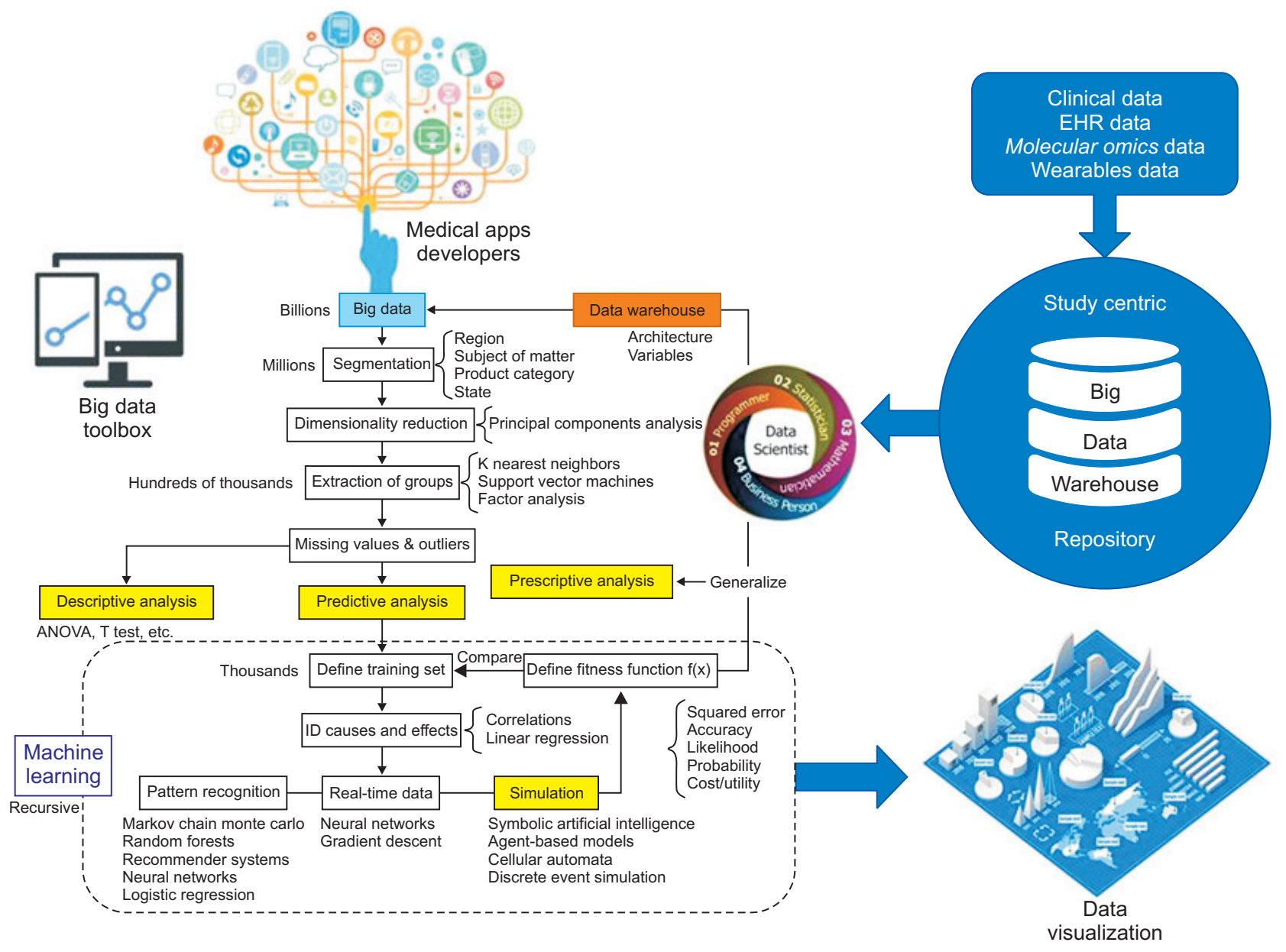

Figure 3. The Centers for Medicare \& Medicaid Services (CMS) data system. 
stand.

(5) Reduced risk: Act on notifications and isolate incidents generated anywhere in the company environment from a single console.

\section{Challenges for Big Data in Healthcare}

The challenges fall into two main categories: fiscal/policy and technology.

Fiscal and policy issues: In a fee-for-service environment, the only way that healthcare practitioners get paid is to have face-to-face encounters with patients. This creates heavy bias against promoting technologies that streamline non-faceto-face interactions. However, as we move away from that model and more towards value-based care, where global risk-based payments are made to delivery organizations (hospitals, patient centered medical homes, accountable care organizations, etc.), then there is more incentive to use new technologies that reduce unnecessary in-office encounters. In such an environment, face-to-face encounters are actually a cost center, not a profit center, and positive health outcomes of populations are rewarded.

Technology issues: The biggest technical barrier to achieving this vision is the state of health data. Created by legacy EHR systems, health data is largely fragmented into institution-centered silos. Sometimes those silos are large, but they are still silos. Exchanging individual records between silos, using increasingly standardized vocabularies (code sets) and message formats (ADT messages, C-CDAs, even FHIR objects), is where much current effort is being directed. But that does not solve the problem of data fragmentation. More and more people in the health information exchange arena are seeing that the next generation of health technology is around aggregating data, not simply exchanging copies of individual records (the traditional query-response approach). Only by collecting the data from many different sources, normalizing that data into a consistent structure, resolving the data around unique patient identifiers as well as unique provider identifiers-only then can the data become truly useful [25].

Aggregated data has two additional advantages. (1) It solves the interoperability problem. Systems and institutions no longer need to build data bridges, and translate how the data is structured between two proprietary systems; everyone instead simply connects to a central standard API "plug." If built right, the aggregated data can be the basis for very effective AI technology. Such technology is very fast (consider Google suggestions as-you-type in a search bar, retrieving suggestions from billions of record options). (2) It is also sufficiently flexible to allow machine learning, and AI will be able to function in a real-time fashion.

\section{New Generation of Digital Health Ad- visors}

Once a data store has been built from many different sources-EHR data, payer data, device and IoT data, patient survey responses, consumer health data-and has been integrated into a unified data structure, then AI can yield meaningful insights. AI, after all, is about pattern recognition, comparing a particular pattern of data around a given individual with similar (not necessarily identical) patterns found elsewhere, and making predictive recommendations based on what happened in those other situations. This is very much what clinicians do when exercising "clinical judgement"-identifying a pattern, taking into account medical problems, medications, labs values, personal and family history, and comparing it to similar patterns from the clinician's experience.

A new generation of "Health Coaches", Tele-Carers or Digital Health Advisors can be trained to make these AI-derived recommendations useful [26]. They need to be easy-to-use, consumer-orientated persons who can connect to the aggregated data store and the AI analytics engines that sit on top of that. They can empower consumers/patients, and reduce the demand burden on clinicians. Will they replace clinicians? No, of course not. But they will help filter the demand to those who truly need to be seen, while empowering patients with real-time, believable and personalized guidance for the more common things in day-to-day life [27].

So what stands in the way of Digital Health Advisors? Policy (how we pay for healthcare) needs to encourage self-care and facilitate healthy behaviors, rather than encourage inoffice doctor visits. And, simultaneously, health data needs to become reorganized in order to empower $\mathrm{AI}$ and drive the emergence of new apps and related technologies. It will be a while before we get there, but we can see the path to that new generation of healthcare technology.

\section{Conclusion}

The mIoT is revamping healthcare services, as people have started using IoT to manage their health requirements. For example, people can use IoT devices to remind them about appointments, changes in blood pressure, calories burnt and much more. One of the best parts of the IoTs in the health- 
care industry is the remote health monitoring system, where patients can be monitored and advised from anywhere. Real-time location services are another major approach IoT offers. By using the service, doctors can easily track device locations, which directly reduces excess time spent. Smartphone usage is increasing rapidly, and people have started using mobile apps for almost everything. When it comes to the healthcare industry, mobile apps can improve communications between patients and doctors over a secured connection.

The primary duty of Digital Health Advisors and the clinicians will be to work collaboratively when the organization is shifting towards IoT-enabled infrastructure. Proper training and feedback are mandatory for better deployment. The traditional method of recording a patient's details, i.e., a pad of paper hanging on the patient's bed, is not going to work anymore, since such records are only accessible to a limited few, and can be lost or scrambled. This is an application where on-field mobile/tablet technology might work, since they offer hassle-free record management on the applications in the device. Health data information will be available in just a tap when information is recorded electronically, once security and privacy issues are met.

\section{Conflict of Interest}

No potential conflict of interest relevant to this article was reported.

\section{References}

1. Zanella A, Bui N, Castellani A, Vangelista L, Zorzi M. Internet of things for smart cities. IEEE Internet Things J 2014;1(1):22-32.

2. Bauer H, Patel $M$, Veira J. The Internet of Things: sizing up the opportunity [Internet]. New York (NY): McKinsey \& Company; c2016 [cited at 2016 Jul 1]. Available from: http://www.mckinsey.com/industries/hightech/our-insights/the-internet-of-things-sizing-up-theopportunity.

3. Kruse CS, Kothman K, Anerobi K, Abanaka L. Adoption factors of the electronic health record: a systematic review. JMIR Med Inform 2016;4(2):e19.

4. Scheen AJ. Precision medicine: the future in diabetes care? Diabetes Res Clin Pract 2016;117:12-21.

5. van Leeuwen N, Swen JJ, Guchelaar HJ, 't Hart LM. The role of pharmacogenetics in drug disposition and response of oral glucose-lowering drugs. Clin Pharmaco- kinet 2013;52(10):833-54.

6. Flores M, Glusman G, Brogaard K, Price ND, Hood L. P4 medicine: how systems medicine will transform the healthcare sector and society. Per Med 2013;10(6):565-76.

7. van Uem JM, Maier KS, Hucker S, Scheck O, Hobert MA, Santos AT, et al. Twelve-week sensor assessment in Parkinson's disease: impact on quality of life. Mov Disord 2016 May 31 [Epub]. http://dx.doi.org/10.1002/ mds.26676.

8. Dzubur E, Li M, Kawabata K, Sun Y, McConnell R, Intille S, et al. Design of a smartphone application to monitor stress, asthma symptoms, and asthma inhaler use. Ann Allergy Asthma Immunol 2015;114(4):341-2.e2.

9. European Commission. Clinical trials - regulation EU No 536/2014 [Internet]. Brussels: European Commission; c2016 [cited at 2016 Jul 1]. Available from: http:// ec.europa.eu/health/human-use/clinical-trials/regulation/index_en.htm.

10. INNT Foundation [Internet]. Amsterdam: INNT Foundation; c2016 [cited at 2016 Jul 1]. Available from: https://www.innit.foundation.

11. MyTomorrows [Internet]. Amsterdam: MyTomorrows; c2016 [cited at 2016 Jul 1]. Available from: https://mytomorrows.com.

12. Tung CE, Su D, Turakhia MP, Lansberg MG. Diagnostic yield of extended cardiac patch monitoring in patients with stroke or TIA. Front Neurol 2015;5:266.

13. Famm K, Litt B, Tracey KJ, Boyden ES, Slaoui M. Drug discovery: a jump-start for electroceuticals. Nature 2013;496(7444):159-61.

14. Cuba-Gyllensten I, Gastelurrutia P, Riistama J, Aarts R, Nunez J, Lupon J, et al. A novel wearable vest for tracking pulmonary congestion in acutely decompensated heart failure. Int J Cardiol 2014;177(1):199-201.

15. Senior M. Novartis signs up for Google smart lens. Nat Biotechnol 2014;32(9):856.

16. MyDario.com [Internet]. Burlington (MA): MyDario. com; c2016 [cited at $2016 \mathrm{Jul}$ 1]. Available from: http:// mydario.com/.

17. SleepBot [Internet]. New York (NY): SleepBot; c2013 [cited at 2016 Jul 1]. Available from: https://mysleepbot. $\mathrm{com} /$.

18. RANKED Health [Internet]. [place unknown: publisher unknown]; [cited at $2016 \mathrm{Jul} \mathrm{1]}$. Available from: http:// www.rankedhealth.com/about/.

19. Canary Health [Internet]. Los Angeles (CA): Canary Health Inc.; c2016 [cited at 2016 Jul 1]. Available from: http://www.canaryhealth.com/. 
20. Sepah SC, Jiang L, Peters AL. Long-term outcomes of a Web-based diabetes prevention program: 2-year results of a single-arm longitudinal study. J Med Internet Res 2015;17(4):e92.

21. Centers for Disease Control and Prevention. Lifestyle change program details [Internet]. Atlanta (GA): Centers for Disease Control and Prevention; c2016 [cited at $2016 \mathrm{Jul} \mathrm{1]}$. Available from: http://www.cdc.gov/diabetes/prevention/lifestyle-program/experience/index. html.

22. HereIsMyData [Internet]. Nijmegen: Radboud University Medical Center; c2015 [cited at 2016 Jul 1]. Available from: http://www.hereismydata.com/.

23. Auffray C, Balling R, Barroso I, Bencze L, Benson M, Bergeron J, et al. Making sense of big data in health research: towards an EU action plan. Genome Med 2016; 8(1):71.
24. Filkins BL, Kim JY, Roberts B, Armstrong W, Miller MA, Hultner ML, et al. Privacy and security in the era of digital health: what should translational researchers know and do about it? Am J Transl Res 2016;8(3):1560-80.

25. Hackl WO. Intelligent re-use of nursing routine data: opportunities and challenges. Stud Health Technol Inform 2016;225:727-8.

26. Foley P, Steinberg D, Levine E, Askew S, Batch BC, Puleo EM, et al. Track: a randomized controlled trial of a digital health obesity treatment intervention for medically vulnerable primary care patients. Contemp Clin Trials 2016;48:12-20.

27. Giraldo-Rodriguez L, Torres-Castro S, Martinez-Ramirez D, Gutierrez-Robledo LM, Perez-Cuevas R. Tele-care and tele-alarms for the elderly: preliminary experiences in Mexico. Rev Saude Publica 2013;47(4):711-7. 